\title{
Forschung und Entwicklung: eine unverhoffte Chance für die GKV
}

\section{Grundsätzliche Bemerkungen zu einem ambitionierten Projekt der Politik}

\section{NORBERT SCHMACKE}

Prof. Dr. med. Norbert Schmacke ist Mitglied der Abteilung Versorgungsforschung des Instituts für Public Health und Pflegeforschung an der Universität Bremen. Er ist darüber hinaus stellvertretendes Unabhängiges Mitglied des Gemeinsamen Bundesausschusses (G-BA) in Berlin. Weitere Mitgliedschaften: Deutsche Gesellschaft für Allgemeinmedizin, Deutsche Gesellschaft für Prävention und Sozialmedizin sowie Netzwerk evidenzbasierte Medizin

\author{
Ein Megasystem wie die Gesetzliche Krankenversicherung \\ ist auf eine kluge Strategie von Forschung und Entwicklung \\ angewiesen. Die im Koalitionsvertrag enthaltenen \\ Bestimmungen zur Finanzierung eines Innovationsfonds \\ und einer längerstreckigen Versorgungsforschung \\ beinhalten Potenziale für eine neue Kultur einer \\ wissenschaftlich gestützten Weiterentwicklung der \\ Versorgungsstrukturen und -angebote. An ausgewählten \\ Beispielen bisheriger Bemühungen um die Evaluation \\ von Systeminnovationen wird dargestellt, wie wichtig \\ es ist, an die Instrumente der Optimierung des Systems \\ hohe methodische Anforderungen zu stellen.
}

Die Absicht der großen Koalition, der GKV die Innovation des Versorgungssystems künftig als systematische Aufgabe in das Pflichtenheft zu schreiben (das ist eine der denkbaren Lesarten) stellt mit Blick auf die Geschichte der bisherigen Reformansätze der Politik eine kleine Sensation dar. Ordnungspolitisch kann man darüber vielleicht streiten, ob diese Verortung lupenrein ist, systemtheoretisch ist dieses Investitionskonzept als solches wenig aufregend: jedes Großunternehmen plant einen festen Posten für F+E (Belitz 2004). Der Hinweis soll fern aller platten Vergleiche etwa zwischen dem Autound dem Gesundheitsmarkt darauf hinweisen, dass es eine systematische Forschung zur Optimierung des Systems der (zer-)gliederten medizinischen Versorgung in Deutschland bislang nicht gibt und dass insofern von einer späten Chance für die GKV gesprochen werden darf. Es geht um nichts Geringeres als die Entwicklung einer neu- en Innovationskultur für das System. Wenn man das so sieht, tritt sogleich die Erkenntnis hinzu, dass kultureller Wandel in aller Regel längere Zeiträume benötigt. Gewohnheiten zu verlassen fällt gerade Megasystemen sehr schwer, der Kurs eines Tankers ist nur auf längere Sicht zu ändern. Ein Teilaspekt dieses Phänomens ist das Verhältnis zwischen Wissenschaft, Praxis und Politik. Ungetrübt ist diese Beziehung von Haus aus nicht, weil Beharrungsvermögen von Praxis, Gestaltungswille von Politik und Erkenntnisinteresse von Wissenschaft unterschiedliche Logiken bedienen (Schmacke 2010). Dies muss mit Blick auf Politik und Wissenschaft so sein, weil ergebnisoffene Forschung nicht nach den Intentionen der Politik schielen kann und weil Politik nicht erwarten kann, dass Forschung Lösungsvorschläge auf dem Silbertablett serviert. Das ist eigentlich leicht zu verstehen, aber es sorgt im Alltag immer wieder für Verwirrungen und endet 
gelegentlich in unfruchtbaren plakativen Beschimpfungen (Typ: Weltfremde Forschung versus dumme Politik). Versorgungsforschung klingt demgegenüber wie ein Schmiermittel zur Überwindung dieser Reibungen. Und dies ist eines der größeren Probleme, die bei der Umsetzung der Vorschläge der großen Koalition zu lösen sind. Forschung lebt von guten Fragestellungen, die mit angemessenen Methoden in adäquaten Zeiträumen untersucht werden. Versorgungsforschung ist nicht deckungsgleich mit der Implementierung gesicherten Wissens in den Alltag, wie gerade von Fachgesellschaften

\section{Die Weiterentwicklung des Systems muss sich künftig auf methodisch gut gemachte Innovationsforschung stützen.}

\section{Forschung zu Modellvorhaben nach SGB V 63ff.}

„Die Krankenkassen oder ihre Verbände haben eine wissenschaftliche Begleitung und Auswertung der Modellvorhaben ... nach allgemein anerkannten wissenschaftlichen Standards zu veranlassen. Der von unabhängigen Sachverständigen zu erstellende Bericht über die Ergebnisse der Auswertung ist zu veröffentlichen" so $\$ 65$ SGB V. Wiethege et al haben 2010 gezeigt, dass dieser Gesetzesauftrag weithin auf seine Umsetzung wartet. Wörtlich: „Die Ergebnisse zeigen ein erhebliches Defizit gemessen an der Intention des Gesetzgebers, die Einführung von Managed-Care in das deutsche Gesundheitssystem durch belastbare Evaluationsergebnisse zu flankieren. Der größte Handlungsbedarf liegt nun in der Beantwortung der gern argumentiert wird. Selbst dort, wo Systeminnovationen auf gut gemachte klinische Studien oder Leitlinien aufsetzen können, bleibt „Anwendungsforschung “ eine komplexe Intervention (Campbell et al 2000, Eccles et al 2003), die selbst wieder Elemente von Grundlagenforschung benötigen kann. Das lässt sich gut am Leitlinienthema zeigen: neben der Tatsache, dass diese häufig unbekannt sind, fällt auch ins Gewicht, dass noch keine Konzepte bekannt sind, wie Widerstände gegen die Befolgung der oft als aufgesetzt erlebten Leitlinien überwunden werden können oder wie etwa auf den berechtigten Einwand der Förderung problematischer Mehrfachverschreibungen bei Vorliegen von Multimorbidität reagiert werden muss. Und auch die hoch zu bewertende Erweiterung von Vertragsmöglichkeiten durch den Gesetzgeber stellt Handlungsfelder für komplexe Interventionen dar, die heute auf dem Boden plausibler Annahmen und nach dem Versuch-und-Irrtum Prinzip betreten werden und bislang fast nicht solide evaluiert werden. Es werden drei ältere Fallbeispiele diskutiert, aus denen Konsequenzen für die Gestaltung der Versorgungsforschung gezogen werden sollten. Andere wichtige Beiträge zur Versorgungsforschung (etwa Analysen zu Defiziten an Hand retrospektiver Studien mittels Routinedaten) bleiben bei dieser Darstellung außer Betracht:
Frage, wie man verhindern kann, dass Evaluationen zu dem unbestritten wichtigen Handlungsfeld nicht durchgeführt oder nicht veröffentlicht werden. Hier sind Gesetzgeber und Krankenkassen gefordert. Die Evaluatoren sollten sich zu einer Veröffentlichung verpflichten. Die Veröffentlichung von Ergebnissen und Erfahrungen der Evaluation von Managed-Care ist notwendige Bedingung für die Weiterentwicklung der auf Managed-Care aufbauenden Strukturen im Gesundheitswesen sowie für die Evaluationsforschung in diesem Bereich. Nur so können Methoden der Evaluation in der Wissenschaft diskutiert und weiterentwickelt werden“. Eine eigene Bewertung publizierter Ergebnisse von Managed Care Projekten kommt zu der Feststellung, dass durchgängig unzureichend belastbare Studiendesigns gewählt worden waren, gleich ob es sich um Ansätze zur Optimierung der Schmerztherapie oder zum Einsatz von Pay For

\section{Der Sonderfall DMP}

Die Einführung der (RSAV-)DMP war von heftigen Kontroversen begleitet, wobei neben standespolitischen auch wissenschaftliche Argumente gegen die Einführung der DMP ins Feld geführt wurde, so von Häussler und Berger, deren Begründungen von Raspe et al. zwar prinzipiell entkräftet werden konnten, Performance handelte (Schmacke 2010). ohne zu diesem Zeitpunkt aber auf deutsche Daten verweisen zu können. Aus wissenschaftlicher Sicht wäre die klare Präferenz gewesen, eine gut durchgeführte cluster-randomisierte RCT zu finanzieren (Gerlach et al 2003), anstatt nach Ausrollen der DMP Programme Second Best Designs einsetzen zu müssen. Die erste anspruchsvolle Evaluationsstudie setzte ein aufwändiges Matched-pairVerfahren ein, es war die ELSID-Studie (ELSID $=$ Evaluation of a Large Scale Implementation of Disease Management Programmes), deren Ergebnisse aufhorchen ließen. Am Ende blieb das Problem, dass sich die Hinweise auf einen Zusatznutzen zwar mehren, dass aber bei strenger Einhaltung methodischer Maßstäbe ein kausaler Zusammenhang nicht behauptet werden kann. Das ist umso bitterer, als die ELSID Studie tatsächlich hoch relevante Effekte auf die Lebenserwartung glaubhaft gemacht hat: die Gesamtsterblichkeit betrug in der DMP-Gruppe 11,3 \%, in der NichtDMP-Gruppe 14,4 \%. Hätte eine Arzneimittelstudie derartige Effekte belegen können, wären ihr Schlagzeilen in allen Zeitungen sicher gewesen. Die zugrunde liegende Beobachtungsstudie erlaubt nun nicht die Schlussfolgerung, dass die Absenkung der Sterblichkeit kausal mit der Einschreibung in das DMP zusammenhing: „Although this reduced mortality cannot be attributed directly to the DMP, extension of the approach within primary care may contribute to increased life expectancy for patients with diabetes“ (Miksch et al 2010) lautete deshalb die begründet zurückhaltende Interpretation des AutorInnengruppe. Die Überlegenheit des Konzepts hausarztgesteuerter DMP bei chronischen Erkrankungen hätte mit anderen Worten auf deutlich besserem Fundament getestet werden können, eine kontrollierte randomisierte Studie wäre fraglos durchführbar gewesen, wenn Politik und Kassen im Schulterschluss nicht auf einer sofortigen flächendeckenden Einführung von DMP bestanden hätten.

Nachdem die Politik an neue DMP denkt, sollte spätestens jetzt Konsens erzielt werden, dass - bei welcher Erkrankung auch immer - eine Aufnahme in das Leistungsversprechen der GKV ohne vorherigen Nachweis der Überlegenheit gegenüber der Regelversorgung wissenschaftlichen Standards nicht stand hält, so groß der politische und öffentliche Druck auch immer sein mögen. 


\section{Integrierte Versorgung}

Das Projekt „Gesundes Kinzigtal“ gilt als Vorbild für die Entwicklung integrierter Versorgung. Es verfolgt das Ziel einer Optimierung der Versorgung durch bessere Schnittstellenorganisation zwischen verschiedenen Leistungserbringern und Sektoren, will eine Verringerung der Morbidität durch gezielte Prävention erreichen und hebt auf einen günstigeren Einkauf externer Produkte (z.B. Medikamente) ab. Ein randomisiertes Setting wurde aus vielerlei Gründen verworfen, wobei besonders interessant ist, dass die Initiatoren des Projektes offenbar meinten, die erwarteten positiven Effekte der Präventionsangebote allen Versicherten zugänglich machen zu müssen (Siegel et al 2011): ein Argument, das sich in der Implementierungsforschung einschließlich der Einführung klinischer Innovationen immer wieder findet und durch Wiederholung nicht besser wird. Zutreffend sagt die Gruppe der Begleitforschung des Kinzigtal Projekts zur Bewertung ihrer tendenziell ja Mut machenden Ergebnisse: „Werden keine randomisierten Studiendesigns angewandt, müssen Abstriche an der internen Validität, d.h.an der Aussagekraft der Studienergebnisse in Kauf genommen werden. Denn selbst wenn eine befriedigende Adjustierung der (nicht-randomisiert bestimmten) Kontrollgruppe in Bezug auf alle bekannten Kovariablen und Confounder gelänge, wäre bei einem nicht-randomisierten Design immer noch damit zu rechnen, dass die ungleiche Verteilung unbekannter Confounder auf Interventions- und Kontrollgruppe zu sys-

\section{Honoriert werden muss in Zukunft auch das Nachdenken über relevante Studien und nicht gut klingende Forschung mit heißer Nadel.}

tematischen Fehlschlüssen führen kann.“ (Siegel et al 2011). Das Kinzigtal-Projekt hätte gerade wegen seines mutigen Ansatzes, nicht noch einmal indikationsbezogene Verträge zu schließen, ein besseres Studiendesign in der Evaluation verdient gehabt.

Die Darstellung könnte bei fehlender Systemkenntnis den Eindruck erwecken, die Kassen seien Hauptverantwortliche der Schwächen der interventionellen In- novationsforschung. Die hier geschilderte Schwachstelle entsteht einfach dadurch, dass der Gesetzgeber die Möglichkeit von Modellvorhaben und die zumindest für die Modelle nach $\mathbb{S} \mathbb{S} 63 \mathrm{ff}$. gebotenen Evaluationen als Gestaltungsoption der Kassen formuliert hatte: freilich ohne die Evaluationsklauseln qualitativ dem EbMZeitalter anzupassen und ohne bislang zu reflektieren - jedenfalls nicht erkennbar dass derartige Evaluationen handfeste Innovationsforschung darstellen und nicht zuletzt auch ihre Finanzierung geregelt werden muss. Die Ärztekammern und das KV-System haben zur Systeminnovation so gut wie keine prospektiven Forschungsbeiträge geleistet, wenn man von interessanten und wichtigen Einzelbeiträge etwa zur Evaluation von Qualitätszirkeln oder Veränderungen der Arbeitsbelastung von Ärztinnen und Ärzten absieht (Fuchs et al 2010). Das KV-System, Kammern und Kassen mit ihren eigenen Instituten haben mit unterschiedlichen Motivationen und Akzenten immer wieder zum Teil grundlegende Beiträge zur Systemanalyse vorgelegt, die zur Hypothesenbildung für interventionelle Forschung und zum Monitoring des Leistungsgeschehens ungemein wichtig sind, aber die methodisch angemessene Testung neuer Versorgungsansätze nicht ersetzen können. Und diese schlichte Botschaft gilt es immer wieder zu kommunizieren.

Die Schlussfolgerungen sind also einfach. Systeminnovationen und die damit zusammenhängende Versorgungsforschung benötigen einen gedanklichen Vorlauf, der aufwändiger ist, als Politik und Sponsoren sich dies herkömmlich vorstellen. Dieser Prozess des Nachdenkens auf dem Weg zu unterschiedlichen, oft gestuften Studiendesign muss in die Kostenund Zeitkalkulationen von Forschungsförderung aufgenommen werden, wenn man nicht riskieren will, Anträge und Konzepte auf den Tisch zu bekommen, die mit heißer Nadel gestrickt sind. Antragsteller müssen begründen, wie sie zu ihrer Themenwahl gekommen sind und warum sie das gewählte Forschungsdesign für angemessen halten. Das klingt für Praktiker und Politiker vermutlich gekünstelt, ist es aber nicht, wie (man entschuldige den nochmaligen Hinweis) die weithin sinnlosen Evaluationsbemühungen zu bisherigen Modellvorhaben schmerzhaft zeigen. In den Anträgen wird auch begründet werden müssen, warum kassenartenübergreifende oder warum kassenartenspezifische Populationen untersucht werden sollen. Dabei taucht unweigerlich immer wieder das Thema auf, wie hoch der methodische Aufwand bei unterschiedlich gewählten Studiendesigns zu veranschlagen ist. Es ist zu wünschen, dass wir in Deutschland den Anschluss an die angloamerikanische Tradition finden, für die letztendliche Testung des Nutzens komplexer Interventionen den Ansatz prospektiver kontrollierter Studien zu favorisieren. Sie sind der Goldstandard und sie sind viel leichter zu verstehen als die exzentrischen mathematischen Bemühungen des seit einiger Zeit wieder bemühten und alles andere als modernen Propensity Score Models.

An relevanten Fragestellungen mangelt es nicht: ob dies der Klassiker Telemedizin für Menschen mit fortgeschrittener Herzinsuffizienz ist oder gestufte Versorgungskonzepte für Menschen mit Depressionen, ob es um die Suche nach qualitätssteigernden und Versorgungslücken füllenden Ansätzen eines neuen Professionenmix geht oder um Interventionen zur Erhöhung der Patientensicherheit. Diskutiert werden könnte auch, ob Deeskalationsstudien in diese Förderwelle passen können, wenn sie mit neuen Behandlungsabläufen gekoppelt sind.

Welche Förderprogramme für diese und andere Fragestellungen denn nun tatsächlich zuständig sind, diese Frage soll am Ende noch kurz beleuchtet werden. Dass die medizinischen und soziologischen Fakultäten der Universitäten Health Services Research nicht schon viel früher als relevantes Forschungsfeld entdeckt haben, ist ein Problem. Dass die klinische Forschung immer noch nicht in der Welt von „Comparative Effectiveness Research“ angekommen ist und damit wesentliche Grundlagen für Versorgungsforschung nach wie vor nicht liefert, ist besonders schmerzhaft. Dass Versorgungsforschung im heutigen Verständnis erst auf Initiative der Krankenkassen als Verbundforschung von GKV, Rehaträgern und BMBF systematischer, allerdings auch mit relativ bescheidenen Volumina angeschoben wurde, ist eine weitere Wahrheit. Dass die DFG im Anschluss hieran mit viel gutem Zureden dazu bewegt werden konnte, Versorgungsforschung auch in ihr Fördertableau aufzunehmen, ist ein weiterer nicht unwichtiger Punkt 
einer solchen Gesamtbetrachtung. Dass die versorgungsforschungsnahe Public Health Förderung zuvor massiv reduziert wurde, ist ein weiterer Puzzlestein dieses wackeligen Gebäudes. Und dass der Staat gigantische Summen in biomedizinische Grundlagenforschung steckt, immer mit dem Versprechen, dadurch übermorgen die großen Rätsel von Demenz oder Krebs zu lösen, sollte im Licht der heutigen Versorgungsprobleme für derartige Gruppen von Kranken Anlass für viele kritische Fragen sein. Vor dem Hintergrund dieses Hindernisrennens steckt die hier angedachte Förderung von Versorgungsforschung aus dem Gesundheitsfonds einen hoch interessanten Kurs ab, der bei ausreichend langem Atem die Versicherten dem Ziel einer qualitativ besseren und wirtschaftlicheren Form der Versorgung ein Stück näher bringen kann. Es könnte zudem gelingen aufzuzeigen, wie gut das Geld für derartige Forschung investiert ist.

\section{Literatur}

Campbell $\mathbf{M}$ et al. Framework for design and evaluation of complex interventions to improve health. BMJ 2000; 321:694-696

Eccles $\mathbf{M}$ et al. Research designs for studies evaluating the effectiveness of change and improvement strategies. Qual Saf Health Care 2003; 13: 47-52

Gerlach FM, Beyer M, Szecsenyi J, Raspe H (2003): Evaluation von Disease-ManagementProgrammen - Aktuelle Defizite, Anforderungen, Methoden. ZaefQ 97, 495-501.

Raspe H, Sawicki P, Schmacke N. Sind vorliegende Disease-Management Programme für Diabetes wirklich unnötig? Gesundheit und Gesellschaft Wissenschaft 2/2004 (April), 4. Jg. 23-31

Häussler B, Berger U:,Bedingungen für effektive Disease-Management-Programme - Analyse, Bewertung und Lösungsansätze für Qualität und Finanzierung“. Nomos Verlag 2004

Miksch A, Laux G, Ose D et al. Is There a Survival Benefit Within a German Primary Care-Based Disease Management Program? American Journal of Managed Care 2010; 16: $49-54$
Schmacke N. Qualitätsmanagement im Gesundheitswesen: der weite Weg der Evidenzbasierung in der Versorgungsforschung. Gesundheit und Sozialpolitik 3/ 2012: 31-35

Schmacke N. Gesundheitsreform: welche Logik zählt? In: Gerlinger T, Kümpers S, Lenhardt U, Wright M. Politik für Gesundheit. Fest- und Streitschriften zum 65. Geburtstag von Rolf Rosenbrock. Huber-Verlag, 2010: 112-121

Schwartz FW, Angerer P (Hrsg.). Arbeitsbedingungen und Befinden von Ärztinnen und Ärzten. In: Fuchs C, Kurth BM, Scriba PC (Reihen-Hrsg.). Report Versorgungsforschung. Deutscher Ärzte-Verlag, Köln 2010

Siegel A, Stößel U, Schubert I, Erle A. Probleme der Evaluation einer regionale integrierten Vollversorgung am Beispiel „Gesundes Kinzigtal" Z. Evid. Fortbild. Qual. Gesundh. wesen (ZEFQ) 105 (2011) 590-596

Wiethege J, Ommen O, Ernstmann, Pfaff H. Transparenz als Voraussetzung für Innovation in der Versorgungsforschung: Defizite am Beispiel der Evaluation von Managed-CareModellvorhaben. Gesundheitswesen 2010; 72: $722-728$

\section{Die Macht der Lobbyisten im deutschen Gesundheitswesen}

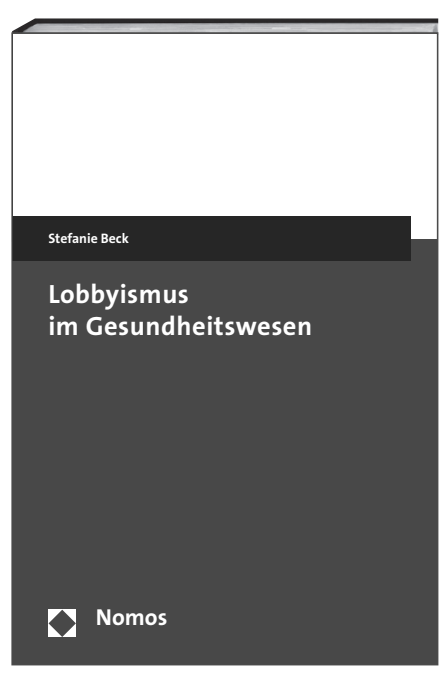

\author{
Lobbyismus im Gesundheitswesen
}

Von Stefanie Beck

2014, 137 S., brosch., 25,-€,

ISBN 978-3-8487-1384-4

www.noms-shop.de/22653

In diesem Buch geht es um die Macht von Lobbyisten im Gesundheitswesen und deren Einfluss auf Politik und Öffentlichkeit. Es analysiert die weitgehend unerforschte und auch intransparente lobbyistische Arbeit der bedeutendsten Lobbyisten im deutschen Gesundheitswesen. Es zeigt, wie die Interessenvertreter ihre Lobbystrategie an die sich ständig verändernden politischen und gesellschaftlichen Strukturen und Prozesse anpassen. Die Organisation der Lobby-Akteure, die Rolle des Informationsmanagement sowie die Art und Weise der Verbreitung von Information werden aufgezeigt. Der Bedeutungsgewinn neuer Medien und nicht hierarchisch kontrollierbarer öffentlicher Kommunikation werden dargestellt und der Lobbyismus begrifflich in den modernen Korporatismus eingeordnet. 